\title{
AGC 226067: A possible interacting low-mass system
}

\author{
E. A. K. Adams ${ }^{1}$, J. M. Cannon ${ }^{2}$, K. L. Rhode ${ }^{3}$, W. F. Janesh ${ }^{3}$, S. Janowiecki ${ }^{3}$, L. Leisman ${ }^{4}$, R. Giovanelli ${ }^{4}$, \\ M. P. Haynes ${ }^{4}$, T. A. Oosterloo ${ }^{1}$, J. J. Salzer ${ }^{3}$, and T. Zaidi ${ }^{2}$ \\ 1 ASTRON, Netherlands Institute for Radio Astronomy, Postbus 2, 7900 AA Dwingeloo, The Netherlands \\ e-mail: adams@astron.n] \\ 2 Department of Physics and Astronomy, Macalaster College, 1600 Grand Avenue, Saint Paul, MN 55105, USA \\ 3 Department of Astronomy, Indiana University, 727 East Third Street, Bloomington, IN 47405, USA \\ ${ }^{4}$ Center for Radiophysics and Space Research, Space Sciences Building, Cornell University, Ithaca, NY 14853, USA
}

Received 30 June 2015 / Accepted 7 July 2015

\begin{abstract}
We present Arecibo, GBT, VLA, and WIYN/pODI observations of the ALFALFA source AGC 226067. Originally identified as an ultra-compact high velocity cloud and candidate Local Group galaxy, AGC 226067 is spatially and kinematically coincident with the Virgo cluster, and the identification by multiple groups of an optical counterpart with no resolved stars supports the interpretation that this systems lies at the Virgo distance $(D=17 \mathrm{Mpc})$. The combined observations reveal that the system consists of multiple components: a central H I source associated with the optical counterpart (AGC 226067), a smaller H I-only component (AGC 229490), a second optical component (AGC 229491), and extended low-surface brightness HI. Only $\sim 1 / 4$ of the single-dish H I emission is associated with AGC 226067; as a result, we find $M_{\mathrm{HI}} / L_{g} \sim 6 M_{\odot} / L_{\odot}$ which is lower than previous work. At $D=17 \mathrm{Mpc}$, AGC 226067 has an H I mass of $1.5 \times 10^{7} M_{\odot}$ and $L_{g}=2.4 \times 10^{6} L_{\odot}$, AGC 229490 (the H I-only component) has $M_{\mathrm{HI}}=3.6 \times 10^{6} M_{\odot}$, and AGC 229491 (the second optical component) has $L_{g}=3.6 \times 10^{5} L_{\odot}$. The nature of this system of three sources is uncertain: AGC 226067 and AGC 229490 may be connected by an Hi bridge, and AGC 229490 and AGC 229491 are separated by only $0.5^{\prime}$. The current data do not resolve the HI in AGC 229490 and its origin is unclear. We discuss possible scenarios for this system of objects: an interacting system of dwarf galaxies, accretion of material onto AGC 226067, or stripping of material from AGC 226067.
\end{abstract}

Key words. galaxies: dwarf - galaxies: ISM - radio lines: ISM

\section{Introduction}

The ALFALFA H I survey offers a census of neutral hydrogen (HI) in the nearby Universe (Giovanelli et al. 2005). Due to its sensitivity and footprint area, ALFALFA has been able to detect rare but extremely interesting objects, including H I sources that probe the extreme limits of systems that are able to form stars. These sources can be coarsely separated into three types: the candidate gas-rich but (nearly) starless Local Group galaxies, called "ultra-compact high velocity clouds" or "UCHVCs" (Giovanelli et al. 2010; Adams et al. 2013, hereafter A13); the low-mass, gas-rich "SHIELD” dwarf galaxies (Cannon et al. 2011); the clearly extragalactic H I detections with no discernible optical counterpart in extant optical surveys, called "AlmostDark" sources (Janowiecki et al. 2015; Cannon et al. 2015, Leisman et al., in prep.).

AGC 226067 is an ALFALFA source that highlights the extreme objects contained within these samples. It was included in the UCHVC catalogs of Giovanelli et al. (2010) and A13 (as HVC274.68+74.70-123) due to its low recessional velocity $\left(c z_{\odot}=-128 \mathrm{~km} \mathrm{~s}^{-1}\right)$, small angular extent $\left(5^{\prime} \times 4^{\prime}\right)$ and isolation from other high-velocity clouds. However, AGC 226067 is projected onto the Virgo Cluster along with at least 14 galaxies located within $3^{\circ}$ and $100 \mathrm{~km} \mathrm{~s}^{-1}$. Two of these systems have Tully-Fisher distances consistent with the distance to Virgo: NGC 4396 (Gavazzi et al. 1999, D = 15.8 Mpc) and IC 3311 (Parnovsky \& Parnowski 2010, $D=21.1 \mathrm{Mpc}$ ). The detection by Bellazzini et al. (2015, hereafter B15) and Sand et al. (2015, hereafter S15) of an optical counterpart to AGC 226067 without resolved stars in ground-based imaging implies $D \gtrsim 3 \mathrm{Mpc}$, negating its identification as an UCHVC and making the association with the Virgo Cluster likely. We assume throughout this work that AGC 226067 is located in the Virgo Cluster at $D=17 \mathrm{Mpc}$. If it is located closer, it is a lower mass system.

In this paper we present global single-dish H I spectra, resolved HI imaging with the Karl G. Jansky Very Large Array (VLA), and deep optical imaging with WIYN/pODI of AGC 226067. Our observations definitively associate the H I with the optical counterpart as a low mass dwarf irregular, analogous to the SHIELD galaxies. Importantly, these data reveal that AGC 226067 is an extended interacting system with multiple components, both $\mathrm{H}$ I and optical.

\section{Data}

\subsection{Single-dish Hı data}

AGC 226067 was originally detected in the Arecibo Legacy Fast ALFA (ALFALFA) H I line survey and is included in the catalogs of Giovanelli et al. (2007) and Haynes et al. (2011); full details of source extraction are given in those works. Due to the lack of an optical counterpart and the low recessional velocity, this source was also included in the UCHVC catalogs of Giovanelli et al. (2010) and A13. In the work of A13, the sources were independently re-measured to ensure that the extended, diffuse emission common to the UCHVCs was properly included, resulting in a flux density that was $15 \%$ higher than given by Haynes et al. (2011). 


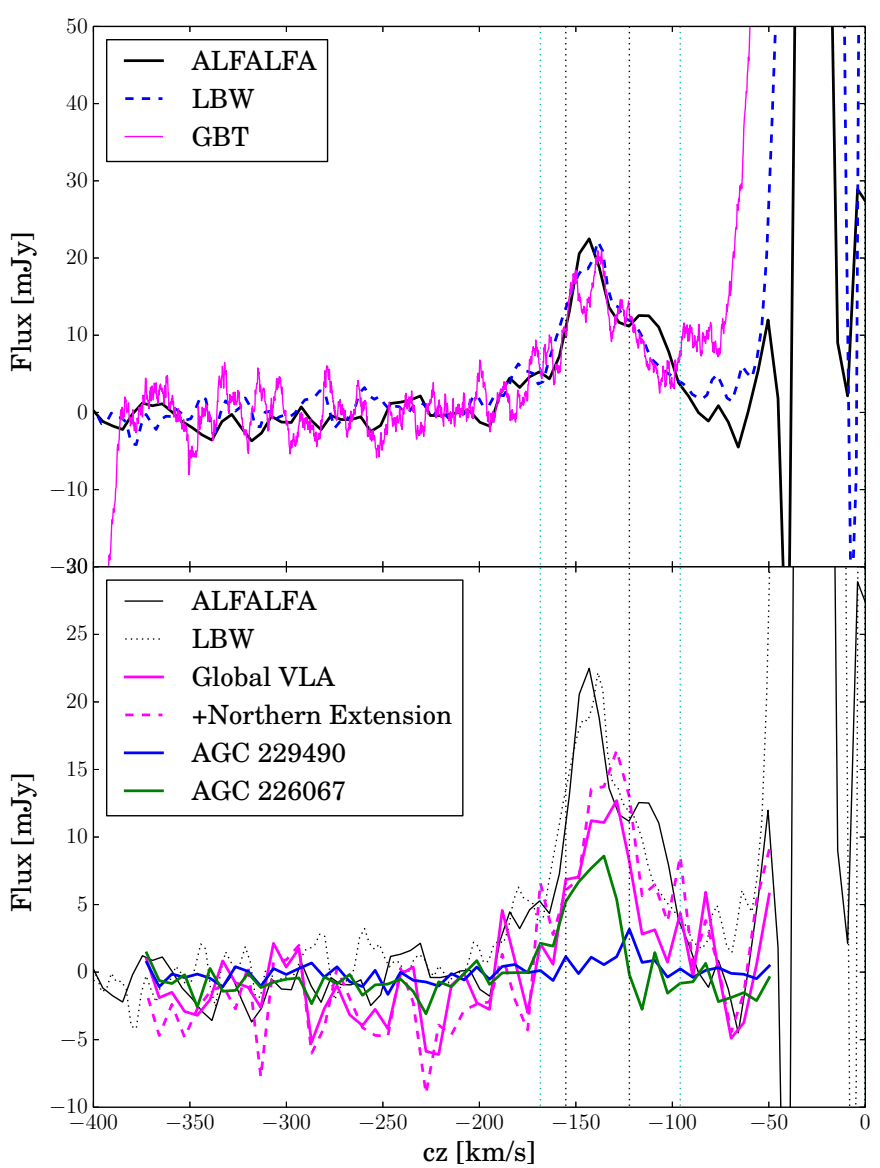

Fig. 1. Top: single-dish spectra for AGC 226067. Both the ALFALFA and LBW spectrum are Hanning smoothed to a velocity resolution of $10 \mathrm{~km} \mathrm{~s}^{-1}$, and the GBT spectrum is boxcar smoothed to a resolution of $5 \mathrm{~km} \mathrm{~s}^{-1}$. Dotted lines indicate the two velocity ranges used for constructing the VLA total H I intensity maps. Bottom: global VLA spectrum, including with the potential northern extension, along with the isolated spectra for AGC 226067 and AGC 229490. The ALFALFA and LBW spectra are included for reference.

AGC 226067 was also observed with both the $L$-band wide receiver (LBW) at Arecibo Observatory (AO; 22 March 2013, program A2752) and the Robert C. Byrd Green Bank Telescope (GBT; 23 October 2012, program GBT/12B-059) as part of programs aimed at confirming UCHVCs. The LBW observation was a 3 min ON-OFF position switching pair, and the GBT observation was a 15 min track in frequency-switching mode. Both spectra were calibrated with observatory-provided routines and the baseline-subtracted spectra are shown in the upper panel of Fig. 1, along with the ALFALFA spectrum from A13.

Table 1 reports the global $\mathrm{H}$ I properties from the single-dish spectra. The LBW spectrum matches the ALFALFA spectrum well but lacks the bump in emission in the red-wing of the profile. Consequently, the measured velocity width and flux density are slightly lower but still consistent with the ALFALFA data. The ALFALFA source size is more extended than the AO beam, so the missing emission could be outside the LBW pointing. However, the GBT beam encompasses the full source extent of AGC 226067. While the GBT spectrum is much noisier, it is a good match to the LBW spectrum, indicating that the lack of red-wing emission may be real and the majority of emission is located within the AO beam. Additionally, all the spectra show low amplitude blue-wing emission, indicating that this may be true emission.
Table 1. Global H I properties.

\begin{tabular}{llll}
\hline \hline Data & $\begin{array}{l}S_{\mathrm{HI}} \\
\mathrm{Jy} \mathrm{km} \mathrm{s}^{-1}\end{array}$ & $\begin{array}{l}c z_{\odot} \\
\mathrm{km} \mathrm{s}^{-1}\end{array}$ & $\begin{array}{l}W_{50} \\
\mathrm{~km} \mathrm{~s}^{-1}\end{array}$ \\
\hline ALFALFA $^{a}$ & $0.92 \pm 0.1$ & $-128 \pm 4$ & $54 \pm 13$ \\
LBW & $0.80 \pm 0.15$ & $-139 \pm 1$ & $48 \pm 6$ \\
GBT & $0.77 \pm 0.18$ & $-139 \pm 2$ & $48 \pm 4$ \\
VLA & $0.47 \pm 0.20$ & $-135 \pm 3$ & $34 \pm 3$ \\
+ Northern extension & $0.68 \pm 0.20$ & $-133 \pm 3$ & $35 \pm 6$ \\
\hline
\end{tabular}

Notes. ${ }^{(a)}$ From A13.

\subsection{VLA HI data}

AGC 226067 was observed in July 2014 with the VLA in D-configuration for 3 one-hour blocks, corresponding to $1.7 \mathrm{~h}$ of on-source integration time. The WIDAR correlator was configured to provide a $4 \mathrm{MHz}$ bandwidth subdivided into 1024 channels, corresponding to a native velocity resolution of $0.82 \mathrm{~km} \mathrm{~s}^{-1} \mathrm{ch}^{-1}$. Standard calibration of the visibility data was done in AIPS, including bandpass, flux and complex gain calibration. Line-free channels were used to remove the continuum emission.

Imaging was done in CASA using Briggs weighting with a robust value of 0.2 ; the resulting clean beam was $47.3^{\prime \prime} \times 43.8^{\prime \prime}$. Clean data cubes binned to a velocity resolution of $6.6 \mathrm{~km} \mathrm{~s}^{-1}$ ( 8 channels) were produced by selecting a clean box that contained all emission from the source and cleaning to 0.5 times the root mean square $(\mathrm{rms})\left(1 \mathrm{mJy} \mathrm{bm}^{-1}\right)$ over the full velocity range of emission ( -168.5 to $-95.9 \mathrm{~km} \mathrm{~s}^{-1}$ ). Total H I intensity (moment zero) maps were created for two different velocity ranges: the spectral extent based on the ALFALFA spectrum ( -168.5 to $\left.-95.9 \mathrm{~km} \mathrm{~s}^{-1}\right)$ and the spectral extent based on the VLA data $\left(-155.3\right.$ to $\left.-122.3 \mathrm{~km} \mathrm{~s}^{-1}\right)$. The spectral extent of the VLA data was determined by spatially smoothing the data cube to a resolution of 100" and selecting channels with contiguous emission. Both H I maps are shown in Fig. 2 and have the same structure: two distinct H I components with evidence for low-level emission connecting them. In addition, the full velocity range map also shows a potential northern extension of emission.

In order to include low level emission when finding the flux density, masks were created by smoothing the moment zero maps to $100^{\prime \prime}$ resolution and clipping at the $3 \sigma$ level. These masks were then applied to original moment zero maps in order to define regions of emission used for calculating the flux density. The recovered flux density for the full spectral range is $0.47 \mathrm{Jy} \mathrm{km} \mathrm{s}^{-1}$. This is $15 \%$ higher than that found for the limited velocity range map, indicating that there is low level emission not clearly evident in the edge channels in the VLA data. In addition, for the full velocity range moment zero map, the northern extension is clearly evident in the smoothed map and is tenuously connected to the rest of the emission at the $3 \sigma$ level. If this is included as part of the source, the total flux density rises to $0.68 \mathrm{Jy} \mathrm{km} \mathrm{s}^{-1}$. We discuss this further in Sect. 3.3.

The source breaks into two distinct H I components; we refer to the main, central source as AGC 226067 and assign the smaller, northern source a new identifier in the Arecibo General Catalog (AGC): 229490. We isolate these two sources by clipping the full spectral range H I map at the $2.5 \sigma$ level. The individual spectra of these sources are shown in Fig. 1, and the H I properties are reported in Table 2. Much of the VLA emission is at low levels surrounding these two sources and is not directly assigned to the individual sources. 

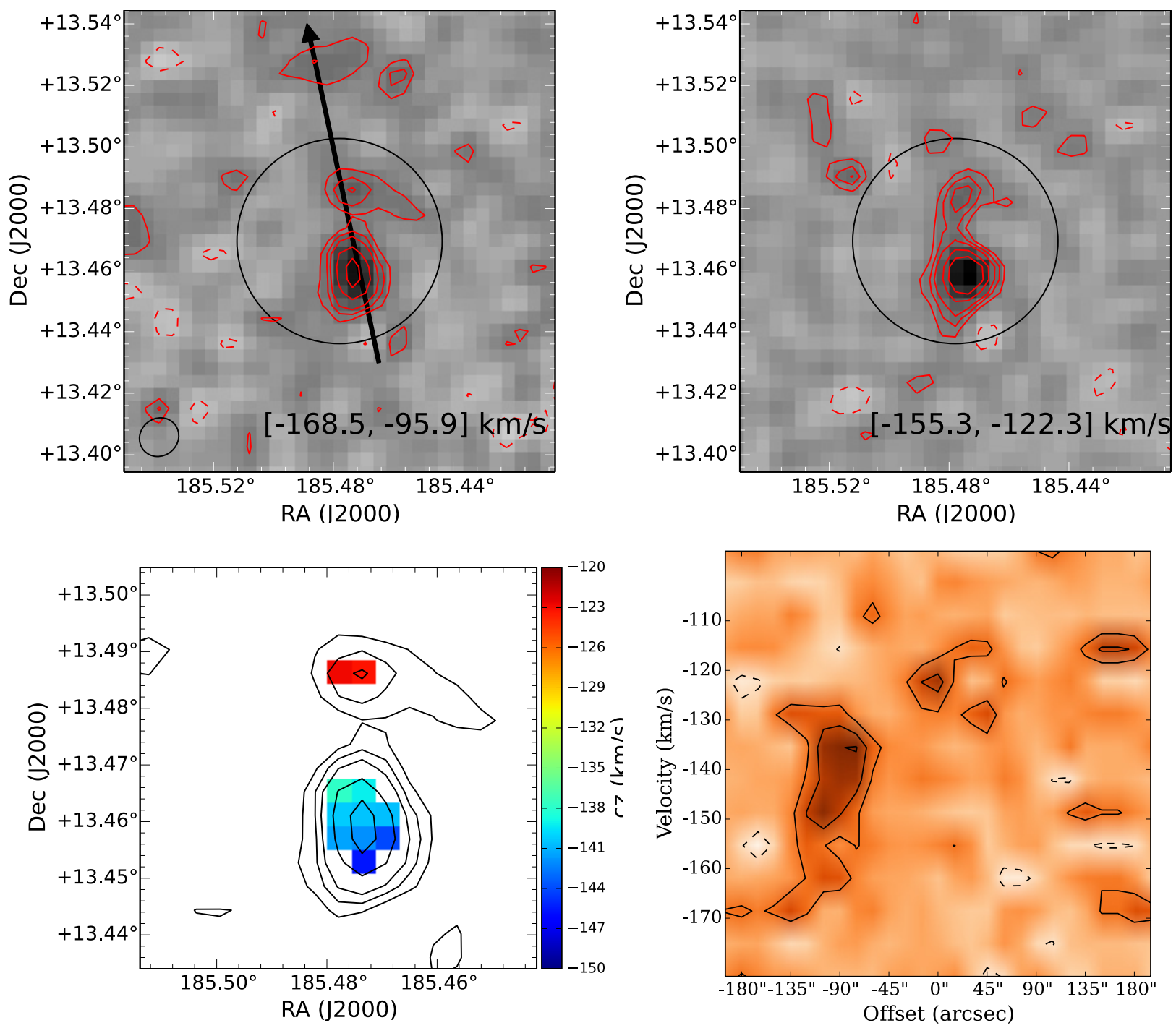

Fig. 2. Top: total intensity H I maps for the two channel ranges. Contours show the $(-2,2,3,4,6,8) \times$ rms value for each map $(0.018$ and $0.015 \mathrm{Jy} \mathrm{km} \mathrm{s}^{-1}$ ). The $4^{\prime} \mathrm{AO}$ beam is shown as the large circle for reference. The synthesized VLA beam is shown as the small circle in the top-left figure. Bottom left: H I intensity contours from the full spectral range H I map (upper left) overlaid on the velocity field from Gaussian fitting. Bottom right: position-velocity slice along the arrow shown in the upper left with contours at $(-2,2,4,6) \times \mathrm{rms}\left(1 \mathrm{mJy} \mathrm{bm}^{-1}\right)$.

We performed a kinematic analysis of the Hi by fitting Gaussian functions to the spectral data cube using XGAUFIT in GIPSY with the requirement that the peak amplitude be $>5 \sigma$ $\left(10 \mathrm{mJy} \mathrm{bm}^{-1}\right)$. The resulting velocity field is shown in Fig. 2. AGC 226067 shows evidence for rotation with an amplitude of $\sim 15 \mathrm{~km} \mathrm{~s}^{-1}$. AGC 229490 is offset by $19 \mathrm{~km} \mathrm{~s}^{-1}$ in central velocity and has narrow fitted velocity dispersions, consistent with the velocity width found in the extracted spectrum but unusual for extragalactic systems. A position-velocity slice bisecting AGC 226067, AGC 229490, and part of the potential northern extension shows that the H I components may be continuous in position-velocity space.

We use these data to place limits on the dynamical masses. The velocity dispersion is an important contribution for these systems and we adopt the expression of Hoffman et al. (1996):

$M_{\mathrm{dyn}}=2.325 \times 10^{5}\left(\frac{V_{\mathrm{rot}}^{2}+3 \sigma^{2}}{\mathrm{~km}^{2} \mathrm{~s}^{-2}}\right)\left(\frac{r}{\mathrm{kpc}}\right) M_{\odot}$.

For AGC 226067, we adopt a rotational velocity of $15 \mathrm{~km} \mathrm{~s}^{-1}$, a velocity dispersion of $9 \mathrm{~km} \mathrm{~s}^{-1}$ based on the Gaussian fitting and $W_{50}$ of the extracted spectrum, and a radius of $4.1 \mathrm{kpc}$ $\left(50^{\prime \prime}\right.$ at $\left.17 \mathrm{Mpc}\right)$, to find a dynamical mass of $4.5 \times 10^{8} M_{\odot}$. For AGC 229490, we assume no rotation and adopt a velocity dispersion of $4 \mathrm{~km} \mathrm{~s}^{-1}$ and a radius of $1.6 \mathrm{kpc}\left(20^{\prime \prime}\right.$ at $17 \mathrm{Mpc}$, slightly smaller than the beam size), giving a dynamical mass estimate of $1.8 \times 10^{7} M_{\odot}$. If the velocity width or H I size of AGC 229490 is larger than revealed by the low signal-to-noise ratio $(\mathrm{S} / \mathrm{N})$ data here, its dynamical mass will also be larger. Conversely, if AGC 229490 is significantly smaller than the beam of these observations, the dynamical mass will decrease.

\subsection{WIYN/pODI data}

AGC 226067 was observed on 14 March 2013 with the partially populated One Degree Imager (pODI; $\sim 24^{\prime} \times 24^{\prime}$ field of view) on the WIYN $3.5 \mathrm{~m}$ telescope ${ }^{1}$. Nine 300 -second exposures were obtained in a dither pattern in the SDSS $g$ and $i$ filters. The data were reduced in the same manner as

1 The WIYN Observatory is a joint facility of the University of Wisconsin-Madison, Indiana University, the University of Missouri, and the National Optical Astronomy Observatory. 
Table 2. Source properties.

\begin{tabular}{llll}
\hline \hline Property & AGC 226067 & AGC 229490 & AGC 229491 \\
\hline RA $(\mathrm{J} 2000)$ & $12: 21: 53.6^{a}$ & $12: 21: 53.8^{a}$ & $12: 21: 55.9^{b}$ \\
Dec $(\mathrm{J} 2000)$ & $+13: 27: 32^{a}$ & $+13: 29: 08^{a}$ & $+13: 29: 04^{b}$ \\
$S_{\mathrm{HI}}\left(\mathrm{Jy} \mathrm{km} \mathrm{s}^{-1}\right)$ & $0.22 \pm 0.06$ & $0.053 \pm 0.04$ & $<0.08$ \\
$c z_{\odot}\left(\mathrm{km} \mathrm{s}^{-1}\right)$ & -142 & -123 & $\ldots$ \\
$W_{50}\left(\mathrm{~km} \mathrm{~s}^{-1}\right)$ & $25 \pm 3$ & $10 \pm 2$ & $\ldots$ \\
$\sigma_{\text {gas }}\left(\mathrm{km} \mathrm{s}^{-1}\right)$ & $9 \pm 3$ & $4 \pm 2$ & $\ldots$ \\
$a \times b(\mathrm{H} \mathrm{I})$ & $105^{\prime \prime} \times 75^{\prime \prime}$ & $45^{\prime \prime} \times 45^{\prime \prime c}$ & $\ldots$ \\
$N_{\mathrm{HI}}\left(\operatorname{atoms~cm}{ }^{-2}\right)$ & $8.6 \times 10^{19}$ & $4.0 \times 10^{19}$ & $<2.8 \times 10^{19}$ \\
$g_{0}{ }^{d}(\mathrm{mag})$ & $20.30 \pm 0.03$ & $\mu_{g}>27.6$ & $22.38 \pm 0.03$ \\
$(g-i)_{0}{ }^{d}(\mathrm{mag})$ & $0.58 \pm 0.04$ & $\ldots$ & $-0.29 \pm 0.08$ \\
$M_{\mathrm{HI}} / L_{g}{ }^{d}\left(M_{\odot} / L_{\odot}\right)$ & $\sim 6$ & $>40^{e}$ & $<14$ \\
\hline \multicolumn{1}{c}{$\mathrm{Distance}-\mathrm{Dependent} \mathrm{Parameters}(D=17 \mathrm{Mpc})$} \\
\hline$r_{\mathrm{HI}}(\mathrm{kpc})$ & 3.7 & $<1.6$ & $\ldots$ \\
$M_{\mathrm{HI}}\left(10^{5} M_{\odot}\right)$ & 150 & 36 & $<50^{f}$ \\
$M_{\mathrm{dyn}}\left(10^{6} M_{\odot}\right)$ & 450 & 18 & $\ldots$ \\
$M_{\mathrm{HI}} / M_{\mathrm{dyn}}$ & 0.03 & 0.2 & $\ldots$ \\
$M_{g}{ }^{d}(\mathrm{mag})$ & -10.85 & $>-7.2^{e}$ & -8.77 \\
$L_{g}{ }^{d}\left(10^{5} L_{\odot}\right)$ & 24 & $<0.85^{e}$ & 3.6 \\
\hline
\end{tabular}

Notes. ${ }^{(a)}$ H I centroid. ${ }^{(b)}$ Optical center. ${ }^{(c)}$ Unresolved by VLA beam. ${ }^{(d)}$ Corrected for Galactic extinction from Schlafly \& Finkbeiner (2011).

${ }^{(e)}$ Based on a 3" aperture and $\mu_{g}>27.6 \mathrm{mag} \operatorname{arcsec}^{-2}$. ${ }^{(f)}$ For an unresolved source with velocity width of $26.4 \mathrm{~km} \mathrm{~s}^{-1}$.

described in Janowiecki et al. (2015): they were run through the QuickReduce data reduction pipeline (Kotulla 2014) using the ODI Pipeline, Portal, and Archive ${ }^{2}$ interface, reprojected onto a common pixel scale, scaled to a common flux level, and stacked. Photometric calibration was done using SDSS stars in the images; errors on the zero points were $<0.02 \mathrm{mag}$. Figure 3 shows the $g$ band image centered on the region of interest.

Figure 4 shows a color image with H I column density contours. The optical counterpart found by B15 (named SECCO 1) and S15 (named ALFALFA-Dw1) is visible and clearly associated with the main HI component, and hence we continue to refer to this source by the original catalog designation, AGC 226067. After carefully masking foreground stars and background galaxies, we measure $g_{0}=20.30 \pm 0.03$ and $(g-i)_{0}=0.58 \pm 0.04$ for an aperture of radius $15^{\prime \prime}$, empirically determined to contain all the emission.

We independently identify the second optical counterpart noted by S15. This source is located near the second H I component (AGC 229490) but offset to the East by 30", so we assign it a unique identification number: AGC 229491. For an aperture of radius $3^{\prime \prime}$ encompassing the blue knot of emission that is AGC 229491, we find $g_{0}=22.38 \pm 0.03$ and $(g-i)_{0}=-0.29 \pm 0.08$.

There is no optical source apparent at the location of the second H I peak (AGC 229490); by measuring the variation in the background levels at the location of AGC 229490, we place $3 \sigma$ surface brightness limits of $\mu_{g}>27.6$ and $\mu_{i}>26.4 \mathrm{mag}$ $\operatorname{arcsec}^{-2}$. If we assume the same optical size as for AGC 229491 (radius of $3^{\prime \prime}$ ), this corresponds to $g>24.0$.

\footnotetext{
2 The ODI Pipeline, Portal, and Archive system is a joint development project of the WIYN Consortium, Inc., in partnership with Indiana University's Pervasive Technology Institute (PTI) and with the National Optical Astronomy Observatory Science Data Management Program.
}

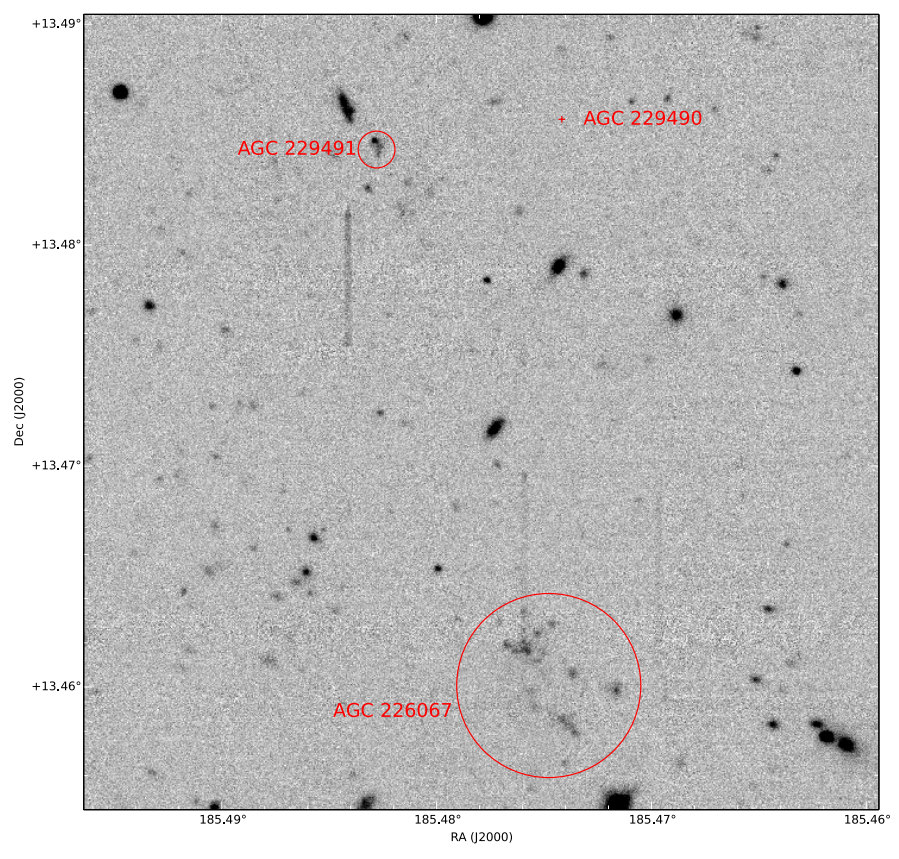

Fig. 3. WIYN/pODI $g$ band image centered on the three sources of interest. The circles indicate the apertures used for photometry for AGC 226067 and AGC 229491, and the red cross the marks centroid of the Hi-only component, AGC 229490.

\section{The sources}

\subsection{AGC 226067: A SHIELD-like dwarf galaxy}

The VLA data allow an unambiguous identification of the $\mathrm{H}$ I in AGC 226067 with the optical counterpart previously identified by B15 and S15. The VLA observations reveal that only part of the H I emission detected by ALFALFA is associated directly with AGC 226067, and the HI mass is thus reduced from values based on the single-dish H I emission by a factor of $\sim 4$ to $1.5 \times 10^{7} M_{\odot}$. Correspondingly, this lowers the $M_{\mathrm{HI}} / L_{V}$ of B15 to $\sim 5$ and the $M_{\mathrm{HI}} / M_{*}$ of $\mathrm{S} 15$ to $\sim 8$. From our pODI data we find $M_{\mathrm{HI}} / L_{g} \sim 6$.

In many ways, AGC 226067 is typical of low mass gas-rich dwarfs, as exemplified by the SHIELD sample of galaxies. The apparent optical extent in our images is $25^{\prime \prime}$, or $\sim 2.1 \mathrm{kpc}$, typical of low mass dwarf galaxies. The total H I mass of $1.5 \times$ $10^{7} M_{\odot}$ and rotational amplitude of $\sim 15 \mathrm{~km} \mathrm{~s}^{-1}$ are consistent with the properties of the SHIELD galaxies (Cannon et al. 2011; McQuinn et al. 2014). However, AGC 226067 is distinguished by its lower surface brightness, its companions, and extended H I disk.

\subsection{AGC 229490 and AGC 229491}

The VLA and deep optical data reveal two additional sources: an H I-only source (AGC 229490) and an optical-only source (AGC 229491). Both are separated from AGC 226067 by $\sim 1.6^{\prime}$ (8 kpc). AGC 229491 was noted by S15, but they were not able to definitively associate it with the AGC 226067 system. The extended Hi nature of this system and the close proximity to AGC $229490\left(0.5^{\prime}\right.$, or $\left.2.5 \mathrm{kpc}\right)$, strongly argue for its physical association with the AGC 226067 system. Treating the AGC 229490 and AGC 229491 as independent sources, we place an upper limit on the HI mass of AGC 229491 of 

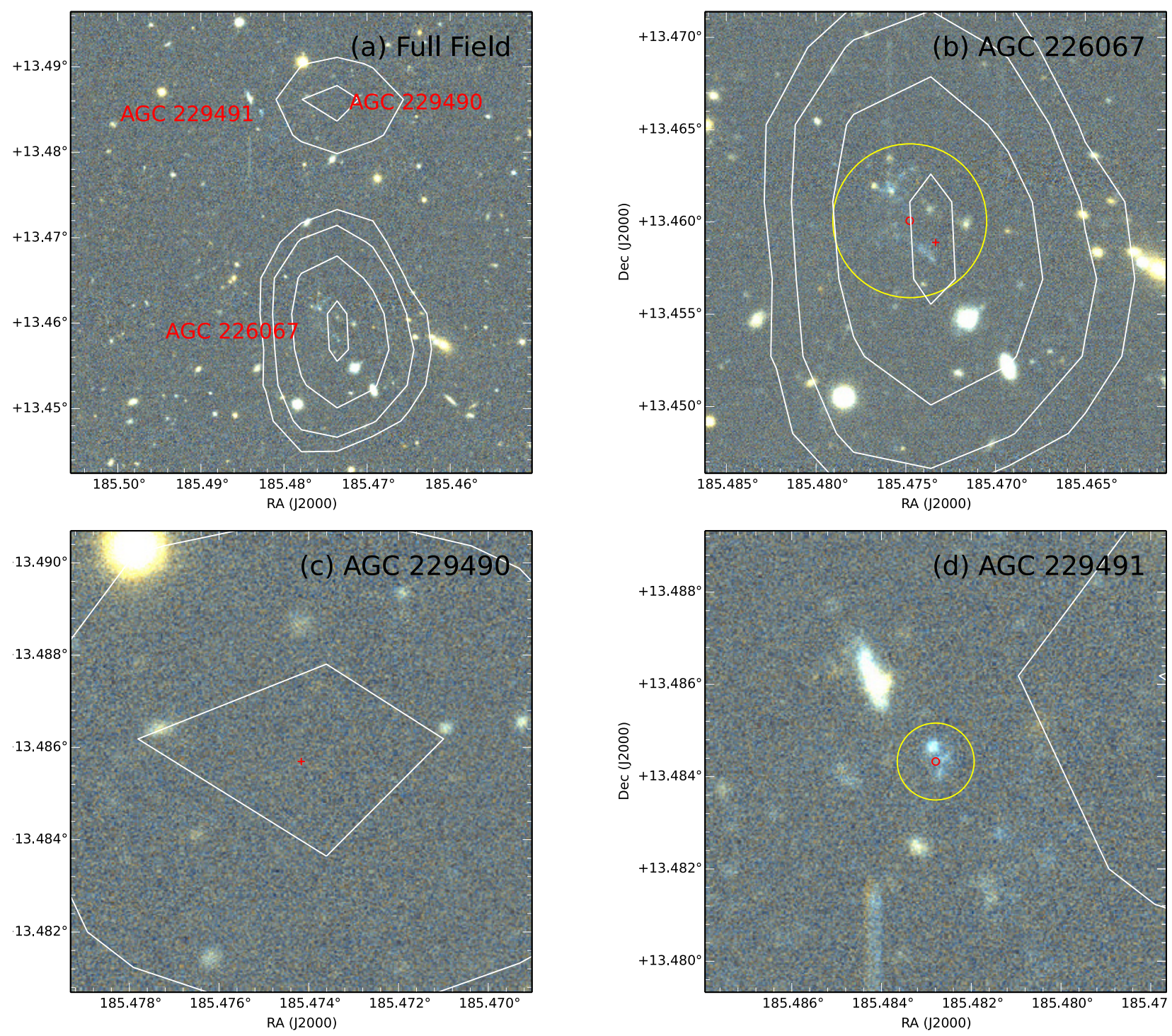

Fig. 4. H I column density contours at $(2.5,3.5,5.5,8) \times 10^{19}$ atoms $\mathrm{cm}^{-2}$ overlaid on a WIYN/pODI color image. Upper left panel: full system, upper right: AGC 226067, lower left: AGC 229490 (H I-only), and lower right: AGC 229491 (optical only). H I centroids are marked with crosses and optical centroids with circles. The yellow circles indicate the apertures used for photometry.

$5.4 \times 10^{6} M_{\odot}$ by assuming it is unresolved and has a velocity extent of $26.4 \mathrm{~km} \mathrm{~s}^{-1}$ (4 channels) $)^{3}$, and an upper limit to the luminosity of AGC 229490 of $L_{g}<0.85 \times 10^{5} L_{\odot}$, assuming the same $r=3^{\prime \prime}$ aperture as for AGC 229491.

Given the close proximity of these two sources, it is plausible that they are directly connected. AGC 229490 could be gas that has been removed from AGC 229491, either through internal or external processes. Alternatively, AGC 229490 and AGC 229491 could be bright peaks of the H I and stellar distribution within the same dark matter halo. Indeed AGC 229490 shows evidence for as extended $\mathrm{H}$ I tail at a low $(2 \sigma)$ level (see Fig. 2). If we consider the two sources associated, we find a global $M_{\mathrm{HI}} / L_{g}$ value of $\sim 10$.

The $M_{\mathrm{HI}} / M_{\text {dyn }}$ value for AGC 229490, while uncertain due to the low resolution and $\mathrm{S} / \mathrm{N}$ of the VLA data, is 0.2 , an unusually high value for dwarf galaxies. This raises the question: is this gas in a dark matter halo or free-floating $\mathrm{H}$ I? Higher resolution

\footnotetext{
3 This upper limit is higher than the measured $\mathrm{HI}$ mass of AGC 229490 as the velocity width of AGC 229490 is smaller than assumed here.
}

and deeper H I data, along with deeper optical data, are needed to fully understand AGC 229490 and AGC 229491 and their relation to AGC 226067.

\subsection{Potential extended emission}

The H I emission seen in the VLA data around AGC 226067 and AGC 229490 only accounts for $\sim 50-60 \%$ of the H I flux seen in the single-dish observations (Table 1), although the VLA data are shallower than the single-dish observations and the reported flux densities are formally consistent within the errors. There is potentially a significant amount of emission $\left(0.21 \mathrm{Jy} \mathrm{km} \mathrm{s}^{-1}\right)$ contained in a northern extension. The VLA spectrum including this extension is shown in Fig. 1, and it does a better job of matching the red edge of emission in the single-dish spectra. However, this emission is low $\mathrm{S} / \mathrm{N}$ and separated from the main body of emission, so it is not clear whether this is real emission.

Given the extended, interacting nature of this system it is quite plausible that there is low-surface brightness emission not recovered by the VLA observations. To test this, we adopt the largest discrepancy of $0.45 \mathrm{Jy} \mathrm{km} \mathrm{s}^{-1}$ (between the 
ALFALFA flux density and the VLA flux density ${ }^{4}$ ). Averaged over an AO beam $\left(4^{\prime}\right)$, this corresponds to a column density of $8.6 \times 10^{18}$ atoms cm $\mathrm{cm}^{-2}$, below the sensitivity of these VLA images $\left(\mathrm{rms}=9.5 \times 10^{18}\right.$ atoms $\left.\mathrm{cm}^{-2}\right)$.

\section{Summary and discussion}

Our combined data reveal that the ALFALFA HI source AGC 226067 is in fact an intriguing system of at least three distinct components plus diffuse $\mathrm{H}$ I emission. Given its kinematic and spatial association with the Virgo Cluster, its most likely distance is $D=17 \mathrm{Mpc}$. The central source, AGC 226067, is a low mass dwarf irregular with extremely low-surface brightness, an extended HI disk, $M_{\mathrm{HI}}=1.5 \times 10^{7} M_{\odot}, M_{\mathrm{HI}} / L_{g} \sim 6$, and $M_{\mathrm{HI}} / M_{\mathrm{dyn}}=0.03$. The H I-only source, AGC 229490 , is possibly connected to AGC 226067 via an H I bridge and has $M_{\mathrm{HI}}=3.6 \times 10^{6} M_{\odot}$ and $M_{\mathrm{HI}} / M_{\mathrm{dyn}} \sim 0.2$. The second optical source, AGC 229491, is extremely blue, consistent with a young stellar population, has $L_{g}=3.6 \times 10^{5} L_{\odot}$, and is offset from AGC 229490 by only $0.5^{\prime}$. The H I data give a strong indication that this system in undergoing some form of interaction. The evidence supporting an interaction includes the putative $\mathrm{H}$ I bridge, a possible HI tail as part of AGC 229490, and the potential extended emission to the north. However, the precise nature of that interaction is uncertain. Deeper and higher resolution data, both $\mathrm{H}$ I and optical, are needed to fully address the origin of this system. Here we take a preliminary look at three possibilities:

- Multiple dwarf galaxy system: AGC 226067, AGC 229490 and AGC 229491 are two or three interacting dwarf galaxies. One possibility is that the AGC 229490/91 system is a single dwarf galaxy that passed through AGC 226067, resulting in the offset of the gas and stars through ram pressure. This is consistent with the potential H I tail of AGC 229490. It is difficult to explain the regular H I appearance of AGC 226067 in this scenario, but deeper H I observations may reveal an irregular H I morphology.

- Accretion onto AGC 226067: AGC 229490 is not gas in a dark matter halo but rather is HI being accreted onto AGC 226067. In this case, AGC 226067 may be undergoing triggered star formation and only recently have developed an optical counterpart, previously being "too shy to shine". However, this scenario does not explain AGC 229491.

- Disruption of AGC 226067: AGC 229490 and AGC 229491 were originally part of AGC 226067, but have been stripped from the central galaxy, either through tidal effects or ram pressure. It is not clear what interaction could have led to the projected orientations of the three components.
It is worth noting that several other systems with extreme $M_{\mathrm{HI}} / \mathrm{L}$ values lie toward the Virgo Cluster, though they are projected further from the cluster center than AGC 226067. This includes HI1225+01, a star-forming dwarf and a dark H I cloud (Chengalur et al. 1995; Salzer et al. 1991), AGC 226178, a dwarf galaxy with a faint optical counterpart offset from a second H I-poor dwarf galaxy (Cannon et al. 2015), and HI1232+20, a system of three H I components of which only one has a detected low-surface brightness counterpart (Janowiecki et al. 2015).

Acknowledgements. We thank M. Johnson for support of the GBT observations, D. Harbeck for support of the WIYN/pODI observations, and the anonymous referee for valuable input. The National Radio Astronomy Observatory is a facility of the National Science Foundation operated under cooperative agreement by Associated Universities, Inc. Based on observations at Kitt Peak National Observatory, National Optical Astronomy Observatory (NOAO Prop. ID:2013A0253; PI:Adams), which is operated by the Association of Universities for Research in Astronomy (AURA) under a cooperative agreement with the National Science Foundation. The ALFALFA work at Cornell is supported by NSF grants AST-0607007 and AST-1107390 to R.G. and M.P.H. and by grants from the Brinson Foundation. K.L.R. and W.F.J. acknowledge support from NSF CAREER award AST-0847109. J.M.C. is supported by NSF grant AST1211683. This research made use of APLpy, an open-source plotting package for Python hosted at http://aplpy.github.com; Astropy, a communitydeveloped core Python package for Astronomy (Astropy Collaboration, 2013); and NASA's Astrophysics Data System.

\section{References}

Adams, E. A. K., Giovanelli, R., \& Haynes, M. P. 2013, ApJ, 768, 77 Bellazzini, M., Magrini, L., Mucciarelli, A., et al. 2015, ApJ, 800, L15 Cannon, J. M., Giovanelli, R., Haynes, M. P., et al. 2011, ApJ, 739, L22 Cannon, J. M., Martinkus, C. P., Leisman, L., et al. 2015, AJ, 149, 72 Chengalur, J. N., Giovanelli, R., \& Haynes, M. P. 1995, AJ, 109, 2415 Gavazzi, G., Boselli, A., Scodeggio, M., Pierini, D., \& Belsole, E. 1999, MNRAS, 304, 595

Giovanelli, R., Haynes, M. P., Kent, B. R., et al. 2005, AJ, 130, 2598

Giovanelli, R., Haynes, M. P., Kent, B. R., et al. 2007, AJ, 133, 2569

Giovanelli, R., Haynes, M. P., Kent, B. R., \& Adams, E. A. K. 2010, ApJ, 708, L22

Haynes, M. P., Giovanelli, R., Martin, A. M., et al. 2011, AJ, 142, 170

Hoffman, G. L., Salpeter, E. E., Farhat, B., et al. 1996, ApJS, 105, 269

Janowiecki, S., Leisman, L., Józsa, G., et al. 2015, ApJ, 801, 96

Kotulla, R. 2014, in Astronomical Data Analysis Software and Systems XXIII, eds. N. Manset, \& P. Forshay, ASP Conf. Ser., 485, 375

McQuinn, K. B. W., Cannon, J. M., Dolphin, A. E., et al. 2014, ApJ, 785,3

Parnovsky, S. L., \& Parnowski, A. S. 2010, Ap\&SS, 325, 163

Salzer, J. J., di Serego Alighieri, S., Matteucci, F., Giovanelli, R., \& Haynes, M. P. 1991, AJ, 101, 1258

Sand, D. J., Crnojević, D., Bennet, P., et al. 2015, ApJ, 806, 95

Schlafly, E. F., \& Finkbeiner, D. P. 2011, ApJ, 737, 103

\footnotetext{
4 Including the northern extension or adopting the LBW/GBT flux density value reduces the discrepancy between the VLA and single-dish flux density values.
} 